\title{
Global Collaboration Around Digital Mental Health: The LAMP Consortium
}

\author{
Rebecca Bilden ${ }^{1} \cdot$ John Torous ${ }^{1}[0$
}

Received: 2 June 2021 / Revised: 2 December 2021 / Accepted: 3 January 2022 / Published online: 18 January 2022

(C) The Author(s), under exclusive licence to Springer Nature Switzerland AG 2022

\begin{abstract}
Despite the great potential, there has been a lack of progress in the development of sharable and scalable tools for digital mental health due to difficulty in reproducibility and clinical application. The LAMP Platform was developed to address this gap by creating a single platform that works for a variety of clinical and research use cases. The study aims to understand how a consortium of clinical and research sites can help onboard, execute, and expand digital health research, software, and use cases. The Division of Digital Psychiatry implemented a formal consortium with goal of expanding the reach of mindLAMP as a digital mental health platform, enabling diverse studies and expanded use cases, and supportint growth of mindLAMP and consortium members' research. The LAMP Consortium has brought together 54 sites from across the world, encouraging collaboration and idea sharing. These sites' locations range from the USA to the Czech Republic to Australia, and apply the many features of LAMP to research, clinical, research and clinical, and industry use. The most popular features were surveys, sharing/viewing data, and GPS passive data collection. A user support network is necessary to encourage research and clinical use of the LAMP Platform. Resources like documentation, an online forum, and newsletters are essential to promote cooperation between many types of sites that is essential to advancing the field of digital mental health.
\end{abstract}

Keywords Mhealth $\cdot$ Mental health $\cdot$ Apps $\cdot$ Digital health $\cdot$ Global health

\section{Introduction}

Interest in digital mental health was expanding before COVID-19 and is now rapidly accelerating as researchers and clinicians seek to improve access and quality of care through leveraging mobile technologies (Torous et al., 2020). However, the sheer amount of digital mental health apps-estimated to be over 10,000 (Torous et al., 2017) and lack of reproducible results in the research literature have hindered clinical adoption and slowed scientific progress (Torous et al., 2018). While the need for thousands of apps and the heterogeneity of research outcomes is understandable in the context of the myriad uses and clinical applications of mobile health technology (Hilty et al., 2021), there are also core common elements that offer opportunity for synergy (Torous et al., 2020). Considering

John Torous

jtorous@bidmc.harvard.edu

1 Division of Digital Psychiatry, Beth Israel Deaconess Medical Center, Harvard Medical School, 330 Brookline Ave, Boston, MA, USA a prior example around computer-based surveys, this problem was identified by a group from Vanderbilt University who created Research Electronic Data Capture (REDCap) to provide a uniform tool that fits a variety of research goals (Harris et al., 2009). To aid in the setup and use for each new site, REDCap founded a consortium that offered support resources (Harris et al., 2009). Towards a similar goal, The Division of Digital Psychiatry at Beth Israel Deaconess Medical Center created LAMP and the LAMP Consortium with the goal of supporting a community around sharing ideas and improving resources in digital mental health (Schueller et al., 2019).

\section{Why Lamp and the Lamp Consortium?}

Digital phenotyping and interventions are costly undertakings that can be difficult to implement, so LAMP was created to meet the mental health field's need (Wasil et al., 2020) for novel data collection for digital phenotyping (via smartphone sensors) (Torous et al., 2019) in tandem with customizable and triggered app interventions (Bidargaddi et al., 
2020). LAMP is an open-source digital mental health platform which allows it to be a foundation for use and collaboration between many types of users. The LAMP consortium was created with a learning health system in mind to enable translational research (Allen et al., 2021) and establish a digital platform for lasting collaboration between patients and mental health professionals from diverse backgrounds.

\section{What is Lamp?}

The acronym LAMP stands for Learn, Assess, Manage, and Prevent, and each category represents a customizable tool for research and/or clinical use. The broad nature of these categories reflects the high degree of personalization and content the app can support across a wide range of use cases (Torous et al., 2019; Liu et al., 2019; Wisniewski et al., 2019; Vaidyam et al., 2020; Henson et al., 2020; Torous et al., 2020; Vaidyam et al., 2019; Gansner et al., 2020; Shvetz et al., 2020; Weizenbaum, 2020; Rodriguez-Villa et al., 2020). The Learn tab enables psychoeducation via resources called "Tips" that includes but is not limited to locally created videos, news links, and articles. The "Assess" tab contains locally created surveys and cognitive assessments (active data) that can be scheduled or taken on demand. The "Manage" tab contains a library of activities designed to reduce symptoms such as mindfulness, journaling, goal tracking, and a hope box. The "Prevent" tab enables return to data and displays information and trends on personal mental health that can be used to assess progress. LAMP can collect multimodal passive data in the form of sensors from a smartphone such as step count, sleep duration, home time, screen time, and other related metrics. A study or clinic can use all tabs and passive data features in harmony or toggle any combination off or on. The circles in Fig. 1 show three sites which have chosen to use different combinations of the available features as an example for flexible use cases supported by LAMP.

The LAMP Platform realizes this potential through three core patient facing components: (1) the app, mindLAMP for Apple, (2) Android and web devices, and (3) the web dashboard. These three components were built to host the tips and activities described above as well as to collect and display data from patients/participants for use in studies and treatments. All components of LAMP were designed with patients/participants in mind and are based off of feedback from a series of ongoing focus groups. Patients have had an active role in the customization of LAMP through the creation of the Learn tab, development of survey questions, gamification of the cognitive test Jewels, development and customization of the Prevent tab, and new sensor types (Torous et al., 2019).

\section{Why a Consortium?}

Health professionals from all backgrounds working together can improve research and clinical care in digital health, and LAMP consortium was created to be a community for them to foster synergy around translational research with the app. By addressing teams' needs around using LAMP (Hilty

\section{LAMP}

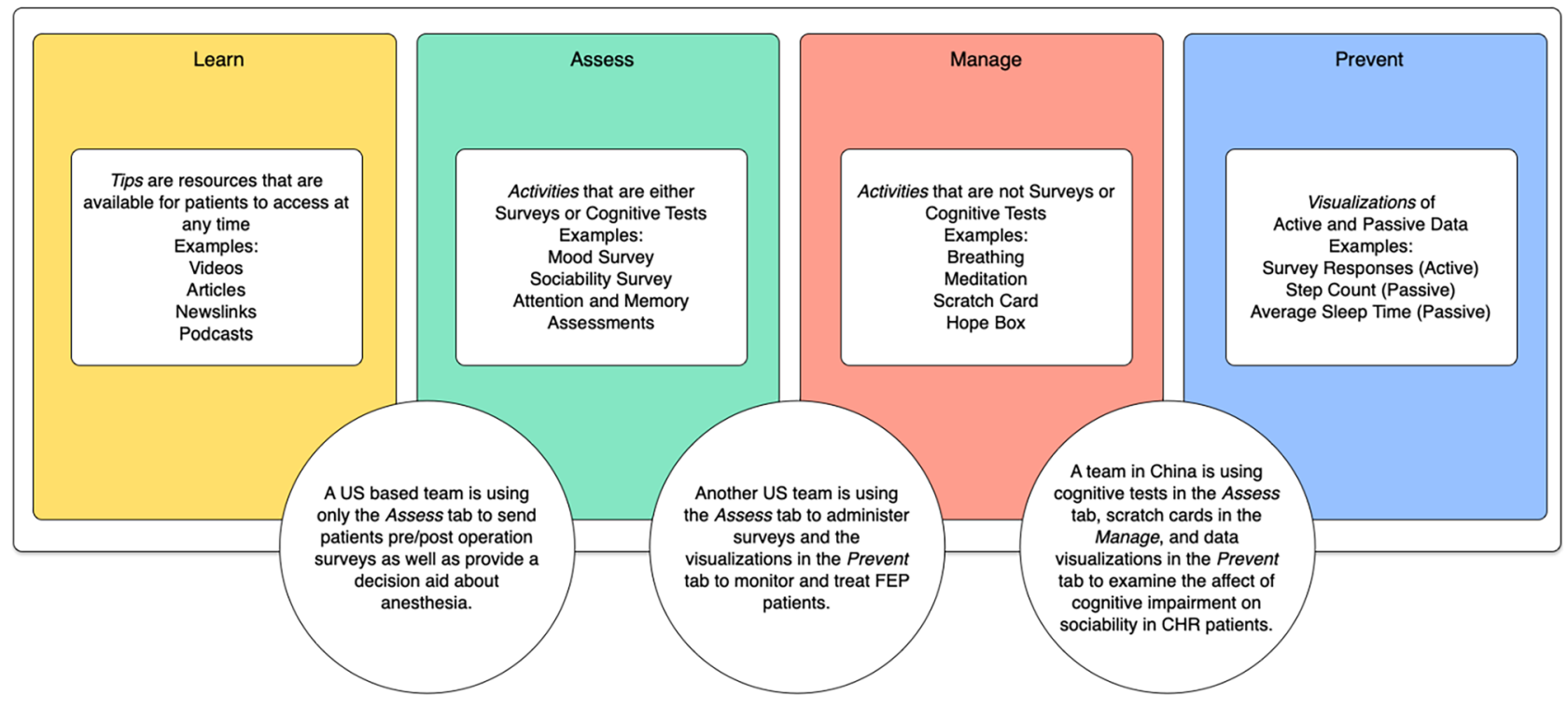

Fig. 1 A schematic function of the features of LAMP 
et al., 2020), designing studies, creating IRBs, customizing the app, deploying the LAMP platform, and analyzing data the consortium aids clinicians, researchers, patients, and technology professionals alike. Data display and collection functions as an integral part of the "LAMP Learning Health System" (Fig. 2) by providing researchers, clinicians, and even patients with valuable insights that can inform study design and new standards of clinical care (Torous et al., 2019).

Sites move between some combination of states at different rates, but all sites will move through state $\mathrm{C}$ regardless of if they are purely clinical, research, or a combination. Table 1 illustrates different examples of current or past consortium member sites within the system.

The LAMP Consortium's focus is on supporting this system and improving the relationship between clinical practices and research by offering a forum for collaborative and iterative scientific progress in digital mental health. In line with this goal, the Division of Digital Psychiatry explored consortium member data to better understand and serve sites' needs and improve patient care.

\section{How - Consortium Management and Collaboration}

The LAMP platform has been open source and freely shared from its inception on GitHub (github.com/BIDMCDigit alPsychiatry). Modeled after a learning health system (Allen et al., 2021), the LAMP Consortium's goal was to create a global network of members who could gather, analyze data, and implement findings in future studies to better clinical care in digital mental health. An onboarding pipeline to track communications and interest in the LAMP Platform was put in place not only to streamline the training process for new members, but also to ensure that members follow this shared improvement methodology. Consortium membership is free, but members must comply with certain ethical, clinical research, and data sharing standards which entail sharing study design and protocols to ensure that use of LAMP is ethical. Members submit a copy of their IRB approval to certify that use of the app is in line with both Apple and Google regulations as well as ensure that all members are following the same ethics standards.

Consortium members are asked to add the same five survey (EMA) questions into their protocol to enable collaboration across studies. All questions are asked and rated on a Likert scale.

- I thought LAMP was easy to use.

- I felt very confident using LAMP.

- I enjoy using LAMP.

- How would you rate your experience with LAMP?

- How would you rate LAMP's usability?

These questions give insight into the way different demographics perceive the usability of the app and dashboard and this information can be used for future platform

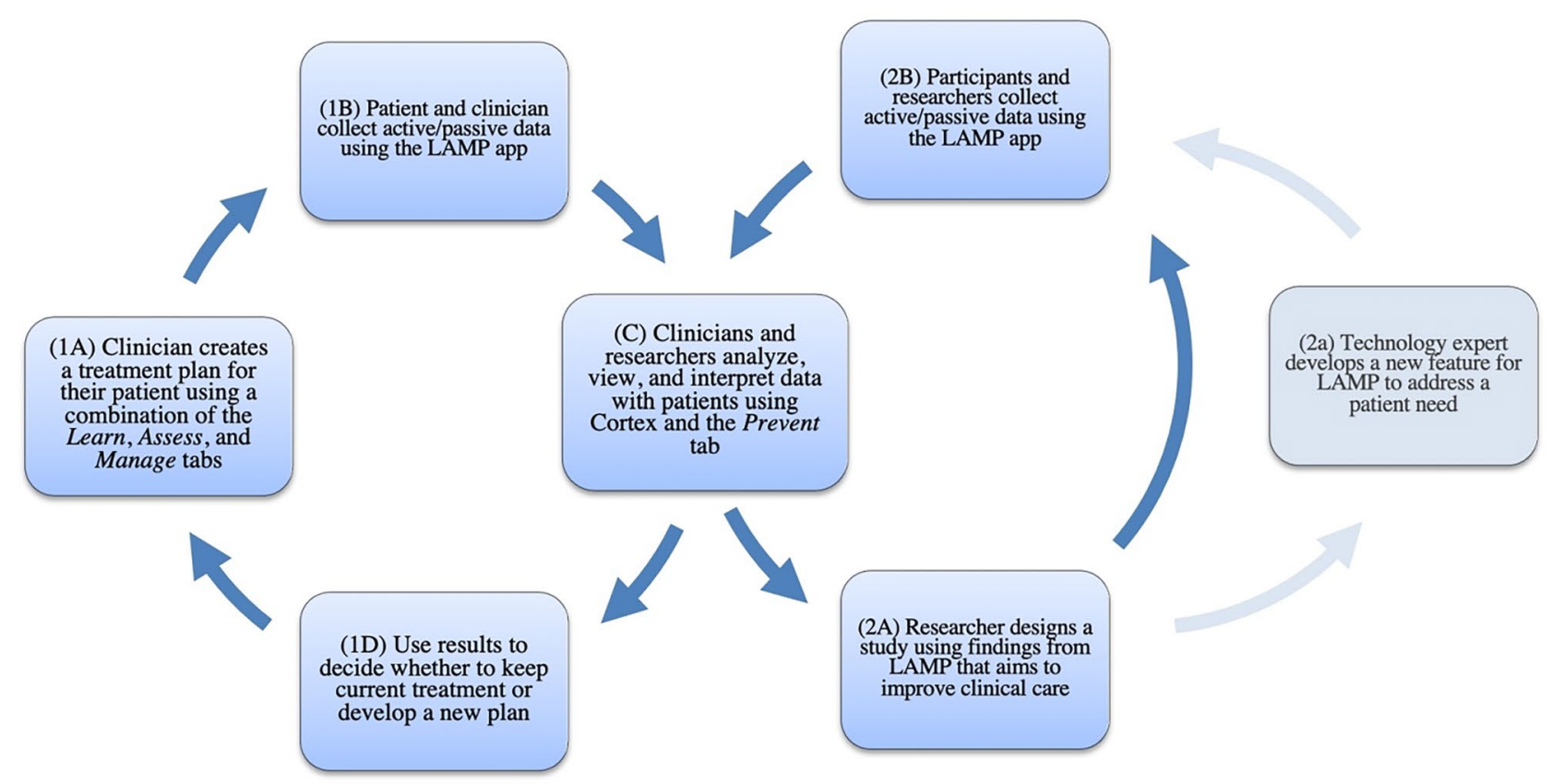

Fig. 2 The states of the "LAMP Learning Health System." The lightest box represents an optional state in the cycle 


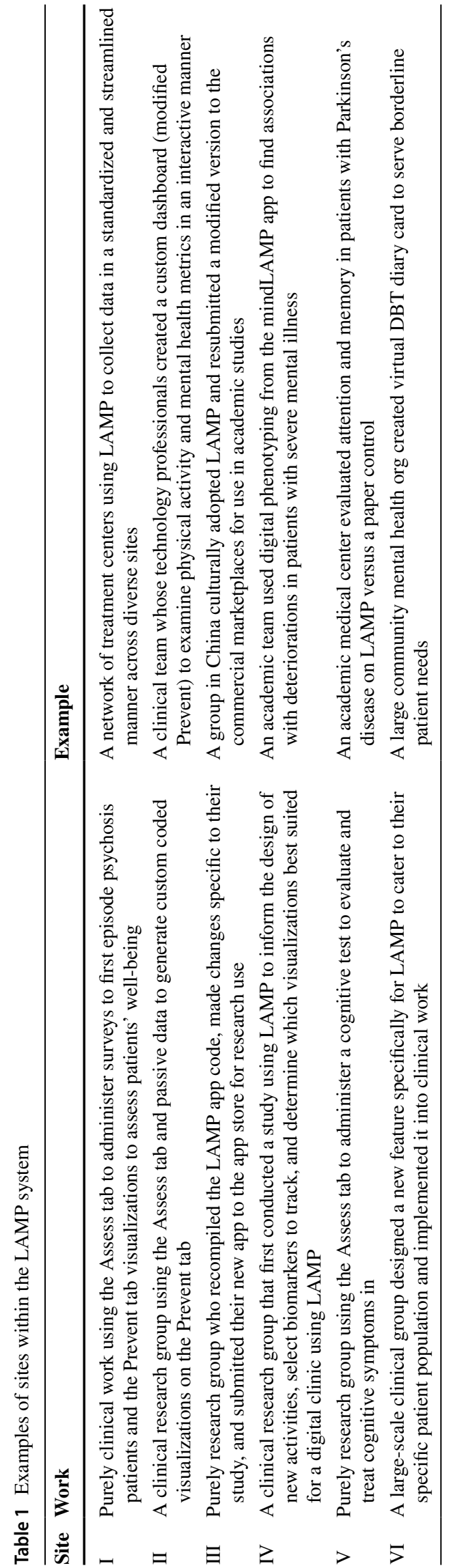

improvements and to normalize data for any subsequent analysis. In addition to these questions, members are able to share non-identifiable data within the consortium because all data collected with LAMP is the structured in the same way (Fig. 3).

Each researcher or clinician has at least one study which contains tips and/or activities. Researchers/clinicians can add participants/patients to a study who then have access to activities which generate active and/or passive data. Patients' data is collected and stored by the LAMP Platform which is HIPPA compliant to guarantee patients both privacy and data security (Di Matteo et al., 2018; Torous et al., 2019). In clinical and research settings, patients/participants must be taught how to use the app and device requirements for passive data collection if applicable (e.g., low-power mode must be kept off). The clinician/researcher works with the patient/participant to administer treatment/gather data and interpret results which foster a relationship that allows for more patient involvement and subsequently improved care (Galvin et al., 2019).

Collecting shared data in a uniform format allows information to be compiled in an anonymized manner at a later date to (1) help assesses site differences and (2) accumulate a global mental health sample for future research that no single-site data could support. The benefit of a uniform data structure is apparent when one site shares their lab's code to process passive data with another consortium researcher; because both sites are using LAMP, the consistent data format allows for the sharing of data processing pipelines.

In addition to the benefits from data sharing, consortium members receive $2 \mathrm{~h}$ of support with setting up LAMP, updates with new features or improved security, access to bimonthly webinars and newsletters, and an online forum to post questions.

Table 2 Distribution of LAMP interest by country

\begin{tabular}{ll}
\hline Country & Count \\
\hline Australia & 2 \\
Brazil & 1 \\
Bulgaria & 1 \\
Canada & 3 \\
China & 1 \\
Czech Republic & 1 \\
England & 2 \\
Estonia & 1 \\
India & 2 \\
Italy & 1 \\
NA & 1 \\
South Korea & 1 \\
Turkey & 1 \\
USA & 36 \\
\hline
\end{tabular}




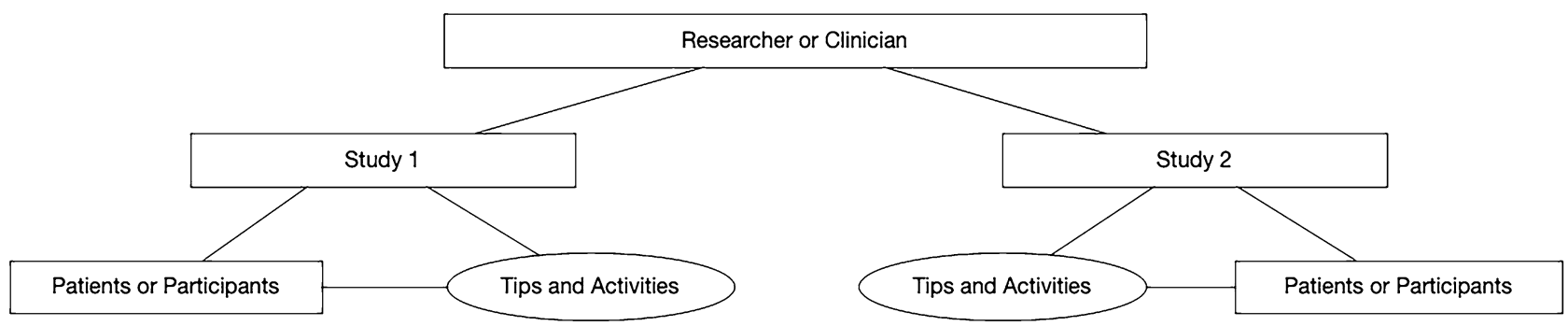

Fig. 3 The structure of data collected with the LAMP Platform

\section{Current Member Metrics}

There are 54 different sites (see Table 2 for country), who have reached out to our team about using LAMP, 48 of which are either using or in the process of planning use. The use cases of these sites are primarily research only (24), but also include clinical (9), both research and clinical (7), and unspecified (8).

The average number of participants in studies or clinics is reported at about 130 people and the average length of a study is about 485 days or 1.3 years. Thirty-seven sites are consortium members, with 9 of the members being self-deploying. There are 3 sites which are self-deploying, independent LAMP users which have chosen to not become members of the consortium, and 12 sites which are currently in the people pipeline to become LAMP users. LAMP consortium members consist almost only of researchers (17) and clinicians (18) with a few industry users (2).

\section{Benefits}

The LAMP Platform is both cost-effective and customizable to diverse needs in the field of digital mental health (Wasil et al., 2020). The LAMP Platform is open-source, so sites only pay to host their data, but more importantly (Bidargaddi et al., 2020), it is customizable and offers multimodal data collection as well as clinical interventions, such as active data collection through surveys and cognitive tests, passive data collection such as device motion sensor data, and data sharing. Figure 4 highlights the diverse interests of LAMP users. The high interest in features like surveys and cognitive tests reflects the popularity of ecological momentary assessments offered (EMA) via smartphones while the interest in ambient light sensor data demonstrates the need for newer, unique features. It is clear from the figure that for a tool such as LAMP to successfully appeal to and be used by sites around the world, it must include a variety of features while continuously expanding its options.

The LAMP Consortium has grown to 54 different sites worldwide engaged with the LAMP Platform in a research and/or clinical setting. The online forum receives about 25 logged in users per day, as well as about 25 anonymous users (see Fig. 5). Consortium members are able to troubleshoot and share ideas using the forum so frequently that sites from around the world have started responding to new questions on the forum - sometimes before the Division of Digital Psychiatry is able. An Australian group responded to a group from Italy who had posted a specific deployment question, and in

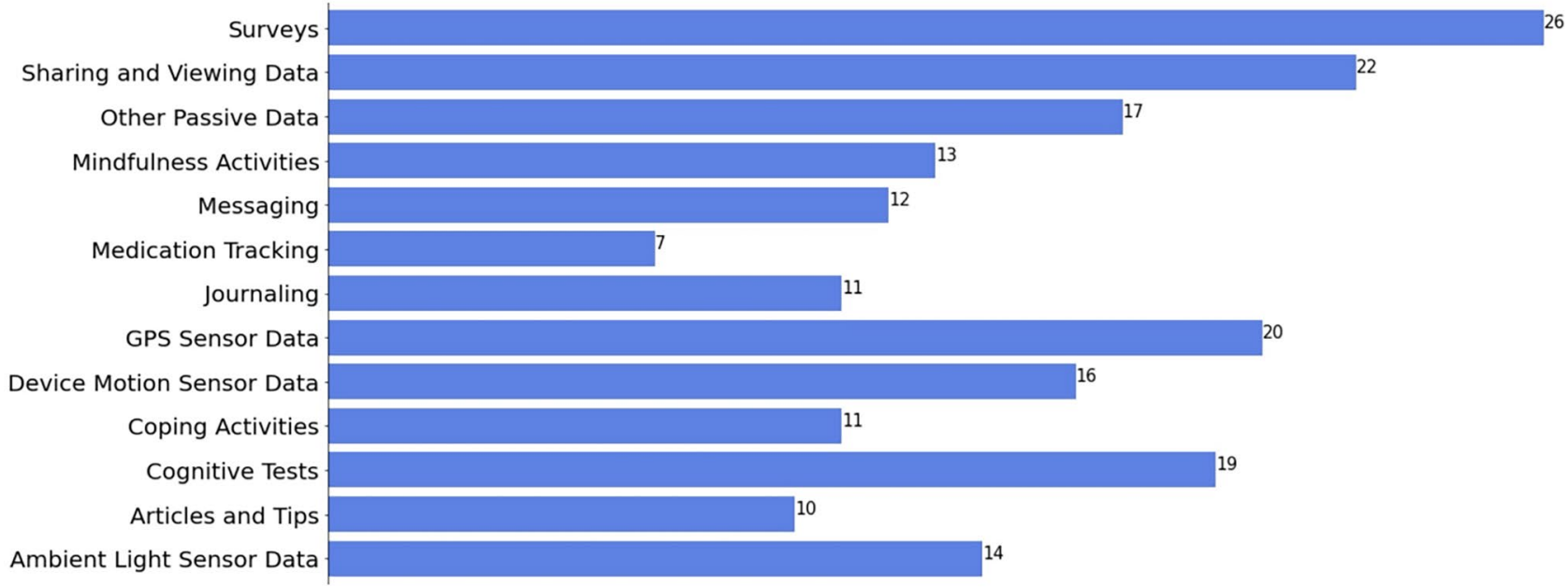

Fig. 4 User interest in LAMP features 


\section{Consolidated Pageviews}

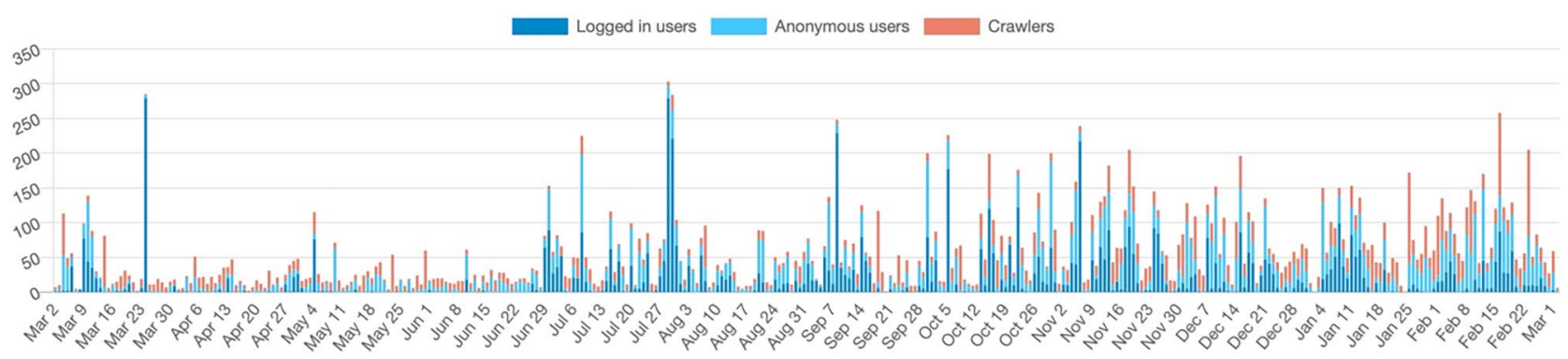

Fig. 5 Average number of forum users per day. Taken from discourse

turn, the Italian group was able to advise a US group about that same issue. Completely self-deploying industry users who are not a part of the consortium have helped members by responding to posts, with one improving five pages of documentation around data science and deployment of the LAMP Platform after going through the steps himself. These changes are still in place and on the LAMP landing page today.

Input from multiple sites is crucial to the development of the LAMP Platform since the primary goal of LAMP was to be a common platform for members to use and improve upon. Having 50+ sites from a variety of backgrounds participating in group bug testing and sending in feature requests provides unparalleled feedback for the Digital Psychiatry team. Documenting issues and solutions that previous sites have found allows new users to make progress, and as a result, sites have contributed by creating mindfulness videos or new cognitive tests. Another notable site contribution was when one group helped the Division of Digital Psychiatry explore a new deployment option with Kubernetes by spending almost $10 \mathrm{~h}$ offering advice around making LAMP easier to deploy, more secure, and therefore more accessible for larger organizations. Other sites have helped translate the app into Spanish and Hindi and adapt it for different cultures, therefore making it more accessible/relevant/useful for future sites.

\section{The Future}

The LAMP Consortium provides researchers and clinicians with a platform that supports study design, technical support through setup, and data collection. In addition to these resources, the LAMP Platform has developed a data analysis pipeline called Cortex which will allow sites to analyze their data in a secure and private way. Incorporating a tool like this allows sites who lack data scientist resources to conduct advanced analysis with their data that they would not have been able to do otherwise. The addition of Cortex will hopefully encourage new and current consortium members to collaborate even further through data sharing and a common tool.

The LAMP Consortium guides users step by step from deployment to research design to data collection and coming soon, analysis. It enables sites to conduct research that may otherwise be unable to perform by supporting through the technical setup and study design process and in return these sites contribute to the consortium by improving LAMP through new feature design, conducting research, and creating new resources for other members' benefit. The LAMP Consortium supports global mental health, reproducible research, and encourages a learning health system approach towards digital mental health. The consortium is a necessary tool to unite mental health professionals and patients around improving research and care in digital mental health.

\section{Declarations}

Conflict of Interest The authors declare no competing interests.

\section{References}

Allen, C., Coleman, K., Mettert, K., Lewis, C., Westbrook, E., \& Lozano, P. (2021). A roadmap to operationalize and evaluate impact in a learning health system. Learning Health Systems, e10258.

Bidargaddi, N., Schrader, G., Klasnja, P., Licinio, J., \& Murphy, S. (2020). Designing m-Health interventions for precision mental health support. Translational Psychiatry, 10(1), 1-8.

Di Matteo, D., Fine, A., Fotinos, K., Rose, J., \& Katzman, M. (2018). Patient willingness to consent to mobile phone data collection for mental health apps: Structured questionnaire. JMIR Mental Health, 5(3).

Galvin, H. K., Petersen, C., Subbian, V., \& Solomonides, A. (2019). Patients as agents in behavioral health research and service provision: Recommendations to support the learning health system. Applied Clinical Informatics, 10(5), 841.

Gansner, M., Nisenson, M., Carson, N., \& Torous, J. (2020). A pilot study using ecological momentary assessment via smartphone application to identify adolescent problematic internet use. Psychiatry Research, 293, 113428. 
Harris, P. A., Taylor, R., Thielke, R., Payne, J., Gonzalez, N., \& Conde, J. G. (2009). Research electronic data capture (REDCap)-A metadata-driven methodology and workflow process for providing translational research informatics support. Journal of Biomedical Informatics, 42(2), 377-381.

Henson, P., \& Torous, J. (2020). Feasibility and correlations of smartphone meta-data toward dynamic understanding of depression and suicide risk in schizophrenia. International Journal of Methods in Psychiatric Research, 29(2), e1825.

Hilty, D. M., Armstrong, C. M., Luxton, D. D., Gentry, M. T., \& Krupinski, E. A. (2021). A scoping review of sensors, wearables, and remote monitoring for behavioral health: Uses, outcomes, clinical competencies, and research directions. Journal of Technology in Behavioral Science, 1-36.

Hilty, D., Chan, S., Torous, J., Luo, J., \& Boland, R. (2020). A framework for competencies for the use of mobile technologies in psychiatry and medicine: Scoping review. JMIR mHealth and uHealth, 8(2), e12229.

Liu, G., Henson, P., Keshavan, M., Pekka-Onnela, J., \& Torous, J. (2019). Assessing the potential of longitudinal smartphone based cognitive assessment in schizophrenia: A naturalistic pilot study. Schizophrenia Research: Cognition, 17, 100144.

Rodriguez-Villa, E., Rauseo-Ricupero, N., Camacho, E., Wisniewski, H., Keshavan, M., \& Torous, J. (2020). The digital clinic: Implementing technology and augmenting care for mental health.

Schueller, S. M., Hunter, J. F., Figueroa, C., \& Aguilera, A. (2019). Use of digital mental health for marginalized and underserved populations. Current Treatment Options in Psychiatry, 6(3), 243-255.

Shvetz, C., Gu, F., Drodge, J., Torous, J., \& Guimond, S. (2020). T99. Harnessing digital technologies to assess and treat cognitive symptoms in schizophrenia. Schizophrenia Bulletin, 46(Suppl 1), S269.

Torous, J., \& Haim, A. (2018). Dichotomies in the development and implementation of digital mental health tools. Psychiatric Services, 69(12), 1204-1206.

Torous, J., Myrick, K. J., Rauseo-Ricupero, N., \& Firth, J. (2020). Digital mental health and COVID-19: Using technology today to accelerate the curve on access and quality tomorrow. JMIR Mental Health, 7(3), e18848.
Torous, J., \& Roberts, L. W. (2017). Needed innovation in digital health and smartphone applications for mental health: Transparency and trust. JAMA Psychiatry, 74(5), 437-438.

Torous, J., \& Vaidyam, A. (2020). Multiple uses of app instead of using multiple apps-A case for rethinking the digital health technology toolbox. Epidemiology and Psychiatric Sciences, 29.

Torous, J., Wisniewski, H., Bird, B., Carpenter, E., David, G., Elejalde, E., \& Keshavan, M. (2019). Creating a digital health smartphone app and digital phenotyping platform for mental health and diverse healthcare needs: An interdisciplinary and collaborative approach. Journal of Technology in Behavioral Science, 4(2), 73-85.

Vaidyam, A., Halamka, J., \& Torous, J. (2019). Actionable digital phenotyping: A framework for the delivery of just-in-time and longitudinal interventions in clinical healthcare. Mhealth, 5.

Vaidyam, A., Roux, S., \& Torous, J. (2020). Patient innovation in investigating the effects of environmental pollution in schizophrenia: Case report of digital phenotyping beyond apps. JMIR Mental Health, 7(8), e19778.

Wasil, A. R., Weisz, J. R., \& DeRubeis, R. J. (2020). Three questions to consider before developing a mental health app. World Psychiatry, 19(2), 252.

Weizenbaum, E. (2020). Cognition "in the wild": Using smartphones to assess cognitive variability in healthy adults and individuals with Parkinson's disease, Dissertation.

Wisniewski, H., Henson, P., \& Torous, J. (2019). Using a smartphone app to identify clinically relevant behavior trends via symptom report, cognition scores, and exercise levels: A case series. Frontiers in Psychiatry, 10, 652.

Publisher's Note Springer Nature remains neutral with regard to jurisdictional claims in published maps and institutional affiliations. 